\title{
Transcriptomic Characteristics of Suboptimal Health Status: Potential Utility of Novel Biomarkers for Predictive, Preventive, and Personalized Medicine Strategy
}

Hao Wang ( $\nabla$ howard.hao.wang@hotmail.com)

Capital Medical University https://orcid.org/0000-0002-9131-0199

Qiuyue Tian

Capital Medical University

Jie Zhang

Capital Medical University

Hongqi Liu

Weifang University

Jinxia Zhang

Capital Medical University

Weijie Cao

Capital Medical University

Xiaoyu Zhang

Capital Medical University

Xingang Li

Edith Cowan University

Lijuan Wu

Capital Medical University

Manshu Song

Edith Cowan University

Yuanyuan Kong

Capital Medical University Affiliated Beijing Friendship Hospital

Wei Wang

Edith Cowan University

Youxin Wang

Capital Medical University

Research 
Keywords: Predictive preventive personalized medicine, Transcriptome profiling, Suboptimal health status, Predictive diagnosis, Novel transcriptomic biomarkers, ATP-binding cassette transporter, Neurodegeneration, Glucokinase regulator

Posted Date: February 25th, 2021

DOI: https://doi.org/10.21203/rs.3.rs-161515/v1

License: (c) (1) This work is licensed under a Creative Commons Attribution 4.0 International License. Read Full License

Version of Record: A version of this preprint was published at EPMA Journal on April 20th, 2021. See the published version at https://doi.org/10.1007/s13167-021-00238-1. 


\section{Abstract \\ Background}

The early diagnosis of Suboptimal Health Status (SHS) creates a window opportunity for the predictive, preventive, and personalized medicine (PPPM) of chronic diseases. Previous studies have observed the alterations in several mRNA levels in SHS individuals. As a promising "omics" technology offering comprehension of genome structure and function at RNA level, transcriptome profiling can provide innovative molecular biomarkers for the predictive diagnosis and targeted prevention of SHS.

\section{Methods}

To explore the potential diagnostic biomarkers, biological functions, and signaling pathways involved in SHS, an RNA sequencing (RNA-Seq)-based transcriptome analysis was firstly conducted on buffy coat samples collected from 30 participants with SHS and 30 age- and sex-matched healthy controls.

\section{Results}

Transcriptome analysis identified a total of 46 differentially expressed genes, in which 22 transcripts were significantly increased and 24 transcripts were decreased in the SHS group. A total of 23 transcripts was selected as candidate diagnostic biomarkers for SHS. Gene Ontology (GO) annotations and Kyoto Encyclopedia of Genes and Genomes (KEGG) pathway enrichment analysis revealed that several biological processes were related to SHS, such as ATP-binding cassette (ABC) transporter and neurodegeneration. Protein-protein interaction (PPI) network analysis identified 10 hub gene related to SHS, including GJA1, TWIST2, KRT1, TUBB3, AMHR2, BMP10, MT3, BMPER, NTM and TMEM98. A transcriptome diagnosis model can distinguish SHS individuals from the healthy controls with a sensitivity of $83.3 \%$ (95\% Confidence Interval (Cl): 73.9\%-92.7\%), a specificity of $90.0 \%$ (95\% Cl: $82.4 \%-97.6 \%)$, and an area under the receiver operating characteristic curve of 0.938 (95\% Cl: $0.882-$ $0.994)$.

\section{Conclusion}

Blood transcripts are potentially objective biomarkers for the SHS diagnosis. These findings determine the potential utility of SHS-related transcriptomic biomarkers for PPPM of chronic diseases.

\section{Introduction}

Predictive, preventive, and personalized medicine (PPPM) is a holistic strategy in healthcare that aims to predict individual predisposition, to provide targeted prevention, and to create personalized treatment [1]. Chronic diseases are usually treated after disease onset, which is a very much delayed approach from the 
perspective of PPPM [2]. Suboptimal health status (SHS) is an intermediate physical state between ideal health and disease, which fits within the paradigm of PPPM [3]. Several studies have suggested that SHS might precede the occurrence of chronic diseases, including type 2 diabetes mellitus (T2DM) $[4,5]$ and cardiovascular diseases (CVD) [6, 7]. As a subclinical stage of chronic disease, the diagnosis of SHS plays a significant role in the targeted prevention and personalized treatment of chronic diseases from the perspective of PPPM [8].

Suboptimal health status questionnaire-25 (SHSQ-25), the most widely used screening tool for SHS, has been developed based on the perceived health complaints and physical symptoms affected by SHS [9]. The SHSQ-25 includes 25 items constituting five dimensions: the immune system, the cardiovascular system, the respiratory system, fatigue, and mental status [9], and it has been used in Caucasians [10], Africans $[4,11]$, and Asian $[6,12]$. To screen objectively diagnostic biomarkers for SHS, several biomarkers, including cortisol [13], relative telomere length [14], intestinal microbiota [15], and metabolites [16], have been investigated. Although previous studies have indicated that multi-omics biomarkers have the potential to diagnose SHS individuals, the underlying mechanisms of SHS remain partially understood.

The transcriptome is a promising "omics" technology involving the identification and quantification of the complete set of transcripts in a specific tissue or cell type. It contains the full information about all RNA transcribed by the genome at a particular developmental stage, and under a certain physiological or pathological condition [17]. Transcriptome analysis not only provides us a better understanding of the human genome at the transcription level, but also gives a comprehensive perspective of genome structure and function [18]. Moreover, it may reveal the key alterations of biological processes that respond to pathogens, diseases, and environmental challenges, thus offering novel molecular biomarkers useful not only for the comprehension of their underlying mechanisms but also for their predictive diagnosis and targeted prevention [19].

Our previous study observed the association between chronic psychosocial stress and SHS, as well as found that decreased mRNA expression of glucocorticoid receptor $a$ is associated with a high level of SHS [20]. The adrenaline and cortisol could impact glucocorticoid receptor expression and splicing [13, 20]. In addition, changes in the transcriptome of circulating immune cells were also observed in patients who suffered from chronic fatigue syndrome, a disease resembling SHS [21]. The biological pathways, including mitochondrial function, oxidative stress, and chronic inflammation are involved in the pathophysiologic mechanism of chronic fatigue syndrome [21]. Tomas-Roig et al. found that changes in mRNA expression, such as mRNA level of dopamine receptor, are associated with long-term psychosocial stress [22]. The association between chronic psychosocial stress and SHS, together with the alterations of mRNA expression in individuals with chronic psychosocial stress, leads to the hypothesis of this study that the alteration of transcriptome profiling might occur in SHS participants and changes in gene expression might be involved in the underlying mechanisms of SHS. 
This study aimed to firstly describe comprehensive transcriptomic biosignature for SHS, and screen objectively diagnostic biomarkers for SHS using RNA-Seq-based transcriptome profiling. In addition, the Gene Ontology (GO) annotation, Kyoto Encyclopedia of Genes and Genomes (KEGG) pathway enrichment analysis and protein-protein interaction (PPI) network analysis of the potential differentially expressed genes (DEGs) were also conducted, so that further understanding of the biological processes involved in SHS and SHS-related chronic diseases was obtained, which proves to be useful for the PPPM of chronic diseases.

\section{Materials And Methods}

\section{Study design and participants}

From September 2017 to November 2017, a case-control study was conducted among a Chinese Han population who received annual health examination at the Student Healthcare Centre of Weifang University. In order to minimize the influence of confounding factors, all the participants were undergraduate students aged 18 to 20 who were living in the university campus apartments. The inclusion and exclusion criteria were described previously in our study [16]. All the participants were required to complete SHSQ-25 in September 2017. After follow-up for 3 months, we assessed their SHS scores for the second time. Participants with SHSQ-25 score $\geq 35$ in both two surveys were selected as cases. Then, age- and sex-matched healthy participants with SHSQ-25 score $<35$ in both two surveys were selected as controls. In total, 30 SHS participants and 30 age- and sex-matched controls were included in the current study.

This study was approved by the Ethics Committee of the Weifang University, Weifang, China. Written informed consent was obtained from each participant at the beginning of the study. The ethics approval was given in compliance with the Declaration of Helsinki.

\section{Data collection}

Demographic characteristics and lifestyle information of participants, including age, sex, ethnicity, smoking, drinking dietary, and sleep duration were collected by questionnaires. The anthropometric measurements, routine biochemical tests, and clinical characteristics were measured as described in our previous study [16]. The physical activity levels, insomnia, anxiety, and depression were measured using the International Physical Activity Questionnaire [23], the Athens insomnia scale [24] the Hamilton anxiety rating scale [25], and the Hamilton depression rating scale [26], respectively.

\section{Blood sample collection and RNA extraction}

Two tubes of blood samples $(5 \mathrm{ml})$ were collected from each participant by venipuncture in the morning after an overnight fasting. One tube of blood sample was collected using a vacuum tube containing polymeric gel to acquire serum, which was used for routine biochemical tests. The other blood sample was collected for RNA extraction. Total RNA was extracted from buffy coat samples of 30 SHS individuals and 30 healthy controls using TRIzol reagent (ThermoFisher Scientific, Waltham, USA). The 
concentration and purity of extracted RNA were measured using Nanodrop 2000 (ThermoFisher Scientific, Waltham, USA). The quality of RNA was assessed using Agilent 2100 Bioanalyzer (Agilent Technologies, Santa Clara, USA). Then, RNA samples were stored at $-80^{\circ} \mathrm{C}$ until RNA-Seq library preparation.

\section{RNA-Seq library preparation and sequencing}

Total RNA samples were quantified using Qubit 2.0 Fluorometer (ThermoFisher Scientific, Waltham, USA). Then, $100 \mathrm{ng}$ of RNA from each sample was used for library preparation using NEBNext Ultra ${ }^{\mathrm{TM}}$ RNA Library Prep Kit for Illumina (New England Biolabs, Ipswich, USA) according to the manufacturer's protocols. Briefly, the mRNA was purified using magnetic beads, and isolated mRNA was reversely transcribed to double-stranded complementary DNA (cDNA). Then, the cDNA libraries were denatured as single-stranded DNA molecules, captured on Illumina flow cells, amplified as clusters, and finally sequenced using an Illumina HiSeq Xten Sequencer (Illumina, San Diego, USA) for 150 bp paired-end reads.

\section{Statistical analysis}

Raw FASTQ files were filtered to remove the adaptors, polyN reads, and low-quality bases using FastQC software. Clean reads were mapped and aligned to the human genome using HISAT2 software, and mapped reads were counted using featureCounts software [27]. Normality distribution of all variables was tested by the Shapiro-Wilk test. Normally distributed continuous variables were reported as mean \pm standard deviation (SD), and non-normally distributed continuous variables were represented as medians and interquartile ranges (IQR). Categorical variables were represented as frequencies and percentages. The differences in categorical variables between the two groups were tested by the Chi-square test or Fisher's exact test. The differences in continuous variables between the two groups were tested by the Student t-test or Mann-Whitney Utest. To identify DEGs related to SHS, mRNA expression profile data in read counts were analysed using the "DESeq2" package in R [28]. The Benjamini-Hochberg method was used to adjust the false discovery rate (FDR). In order to identify the biological functions and pathways in which DEGs were enriched, GO and KEGG enrichment analyses were performed using the "clusterProfiler" package in $\mathrm{R}$ [29]. To further explore the interaction among the DEGs, a PPI network of DEGs was constructed using the Search Tool for the Retrieval of Interacting Genes (STRING) database [30]. The top 10 genes ranked by degree were selected as hub genes using the cytoHubba application [31], while the Cytoscape software 3.8.1 (National Institute of General Medical Sciences, Bethesda, USA) was used to visualize the PPI network. Multivariate binary logistic regression was used to construct transcriptome diagnosis models for SHS. Receiver operating characteristic (ROC) curves and the area under the curve (AUC) were used to assess the diagnostic performance of the models.

Data analysis was performed using SPSS 25.0 (IBM Corporation, New York, USA) and R 4.0.2 [32]. All reported $P$ values were two-tailed, and $P<0.05$ was considered statistically significant.

\section{Results}




\section{Characteristics of participants}

The average ages of SHS and control groups were 19.03 and 18.87 years, respectively. Higher SHS, insomnia, anxiety, and depression scores were observed in the SHS group (All $P<0.05$ ). Aside from these, there were no statistically significant differences observed between the SHS and control groups in terms of the other variables (All $P>0.05$ ). The characteristics of participants were summarized in Supplementary Table 1.

\section{Identification of potential transcriptomic biomarkers}

A total of 46 DEGs were identified between SHS and control group, including 22 upregulated genes and 24 downregulated genes in SHS group, based on the criteria of $P$ value $<0.05$ and the fold change $>2$ (Fig. 1). The detailed list of 46 DEGs were presented in Supplementary Table 2. The AUC values were calculated to assess the diagnostic accuracy of 46 candidate transcripts (Supplementary Table 3). As shown in Table 1, 23 transcripts with statistically significant AUC values were selected as the candidate biomarkers for SHS. 
Table 1

Diagnostic accuracy of candidate transcripts

\begin{tabular}{|c|c|c|c|c|c|}
\hline Gene Symbol & Sensitivity & Specificity & AUC & $95 \% \mathrm{Cl}$ & $P$ value \\
\hline Diagnostic Model & $83.3 \%$ & $90.0 \%$ & 0.938 & $(0.882-0.994)$ & $<0.001$ \\
\hline$A B C A 4$ & $50.0 \%$ & $83.3 \%$ & 0.667 & $(0.528-0.806)$ & 0.026 \\
\hline AMHR2 & $60.0 \%$ & $83.3 \%$ & 0.703 & $(0.569-0.838)$ & 0.007 \\
\hline BMPER & $50.0 \%$ & $90.0 \%$ & 0.705 & $(0.572-0.838)$ & 0.006 \\
\hline CAMK2B & $56.7 \%$ & $76.7 \%$ & 0.682 & $(0.545-0.819)$ & 0.016 \\
\hline CCL3 & $73.3 \%$ & $56.7 \%$ & 0.660 & $(0.520-0.800)$ & 0.033 \\
\hline CYP2W1 & $63.3 \%$ & $73.3 \%$ & 0.691 & $(0.555-0.828)$ & 0.011 \\
\hline DNAJC12 & $73.3 \%$ & $70.0 \%$ & 0.740 & $(0.612-0.868)$ & 0.001 \\
\hline$E M X 1$ & $56.7 \%$ & $70.0 \%$ & 0.649 & $(0.510-0.789)$ & 0.047 \\
\hline$E P G N$ & $33.3 \%$ & $96.7 \%$ & 0.662 & $(0.523-0.801)$ & 0.031 \\
\hline GCKR & $50.0 \%$ & $86.7 \%$ & 0.693 & $(0.558-0.828)$ & 0.010 \\
\hline HJV & $50.0 \%$ & $80.0 \%$ & 0.657 & $(0.518-0.796)$ & 0.036 \\
\hline$H R N R$ & $73.3 \%$ & $33.3 \%$ & 0.711 & $(0.580-0.842)$ & 0.005 \\
\hline LCN8 & $83.3 \%$ & $50.0 \%$ & 0.683 & $(0.549-0.818)$ & 0.015 \\
\hline NKAIN4 & $43.3 \%$ & $90.0 \%$ & 0.650 & $(0.510-0.790)$ & 0.046 \\
\hline$N P W$ & $50.0 \%$ & $86.7 \%$ & 0.674 & $(0.537-0.812)$ & 0.020 \\
\hline OR10AD1 & $73.3 \%$ & $60.0 \%$ & 0.687 & $(0.551-0.822)$ & 0.013 \\
\hline PCDHGB4 & $73.3 \%$ & $53.3 \%$ & 0.678 & $(0.542-0.814)$ & 0.018 \\
\hline RANBP17 & $66.7 \%$ & $73.3 \%$ & 0.709 & $(0.575-0.842)$ & 0.005 \\
\hline SLC12A1 & $60.0 \%$ & $63.3 \%$ & 0.652 & $(0.513-0.792)$ & 0.043 \\
\hline TBC1D3 & $70.0 \%$ & $70.0 \%$ & 0.697 & $(0.562-0.831)$ & 0.009 \\
\hline TMEM211 & $53.3 \%$ & $83.3 \%$ & 0.653 & $(0.513-0.793)$ & 0.041 \\
\hline TUBB3 & $60.0 \%$ & $70.0 \%$ & 0.679 & $(0.543-0.815)$ & 0.017 \\
\hline TWIST2 & $76.7 \%$ & $56.7 \%$ & 0.695 & $(0.561-0.829)$ & 0.009 \\
\hline
\end{tabular}




\section{GO enrichment analysis}

To investigate the biological functions related to SHS, GO enrichment analysis of 46 DEGs was performed. Significantly enriched terms were those with a $P$ value $<0.05$ and gene count $\geq 2$. The results revealed that the 22 upregulated DEGs were significantly enriched in intermediate filament, contractile fiber, intermediate filament cytoskeleton, and apical plasma membrane in terms of cellular component (CC) (Fig. 2A). Regarding biological process (BP), the upregulated DEGs were enriched in adult heart development, organophosphate ester transport, male gonad development, development of primary male sexual characteristics, male sex differentiation, gonad development, protein heterotetramerization, development of primary sexual characteristics, regulation of cardiac muscle cell proliferation, and cardiac muscle cell proliferation (Fig. 2B). Under molecular function (MF), the upregulated DEGs were enriched in ATPase-coupled transmembrane transporter activity, primary active transmembrane transporter activity, lipid transporter activity, and growth factor activity (Fig. 2C). For the downregulated DEGs, significant enrichment was observed in anchored component of membrane and vacuolar lumen regarding CC (Fig. 2D). In terms of BP, the downregulated DEGs were enriched in negative regulation of bone morphogenetic proteins (BMPs) signalling pathway, positive regulation of extracellular signal-regulated kinase-1 (ERK1) and ERK2 cascade, negative regulation of ossification, gliogenesis, regulation of cellular response to growth factor stimulus, regulation of ERK1 and ERK2 cascade, regulation of BMP signalling pathway, ERK1 and ERK2 cascade, cellular transition metal ion homeostasis, and regulation of gliogenesis (Fig. 2E). For MF, the downregulated DEGs were enriched in protein serine/threonine kinase activity (Fig. 2F).

\section{KEGG enrichment analysis}

To explore the biological pathways related to SHS, KEGG enrichment analysis of DEGs was performed. As shown in Fig. 3A, the upregulated DEGs were significant enrichment in ATP-binding cassette (ABC) transporters. In addition, KEGG enrichment analysis identified three significant pathways of the downregulated DEGs, including proteoglycans in cancer, Parkinson disease, and neurodegeneration (Fig. 3B).

\section{PPI network analysis and hub gene recognition}

In order to provide a comprehensive view of potential functional relationships of DEGs, the 22 upregulated genes and 24 downregulated genes were mapped by the STRING database to establish a PPI network (Fig. 4). Protein pairs with a combination score $>0.15$ were selected in the PPI network. The top 10 genes with the highest degree of connectivity were defined as critical hub genes (Fig. 5), including gap junction protein a1 (GJA1), twist family basic helix-loop-helix transcription factor 2 (TWIST2), keratin 1 (KRT1), tubulin $\beta 3$ (TUBB3), anti-mullerian hormone receptor type 2 (AMHR2), bone morphogenetic protein 10 (BMP10), metallothionein 3 (MT3), bone morphogenetic protein binding endothelial regulator (BMPER), neurotrimin (NTM), and transmembrane protein 98 (TMEM98).

\section{Predictive diagnosis model for SHS}


Multivariate binary logistic regression analysis was conducted to construct a diagnostic model based on the transcripts of 10 hub genes. The model based on candidate transcripts was as follows: Logit ( $p=$ $\mathrm{SHS})=0.137-66.878 \times(B M P E R \mathrm{mRNA})+29.328 \times($ AMHR2 $\mathrm{mRNA})$. The model yielded a sensitivity of 83.3\% (95\% Cl: $73.9 \%-92.7 \%)$, a specificity of $90.0 \%$ (95\% Cl: $82.4 \%-97.6 \%)$, and an AUC of 0.938 (95\% Confidence Interval (Cl): 0.882-0.994, $P<0.001$ ) (Table 1 \& Fig. 6).

\section{Discussion}

As a reversible stage in advance of chronic disease, SHS proposes a new effective conception for population risk stratification under the perspective of PPPM. In addition, identifying key biological pathways relevant to the progression of SHS towards chronic diseases is considered as a novel and viable strategy for predictive diagnosis, targeted prevention and personalized therapy of chronic disease. With the emergence of RNA-Seq-based technologies, transcriptome profiling plays a significant role in deciphering gene expressions on RNA level and identifying molecular biomarkers. In the present study, 46 genes are differentially expressed between individuals with SHS and individuals with ideal health status. GO annotations and KEGG pathway enrichment analysis also revealed that several biological processes, such as $A B C$ transporter and neurodegeneration, were related to SHS, and 10 hub genes with the highest degree of connectivity were identified. In addition, the AUC of the predictive diagnostic model based on transcriptomic biomarkers was 0.938 (95\% Cl: 0.882-0.994). These findings suggest that blood transcripts are potentially objective biomarkers for the SHS diagnosis. These transcriptomic biomarkers provide better insight into the critical genes associated with SHS and a deeper understanding of its biological processes.

In the present study, significantly lower level of GCRK mRNA was found in individuals with SHS. Glucokinase regulator protein, encoded by glucokinase regulator $(G C K R)$ gene, is a hepatocyte-specific regulatory protein that inhibits glucokinase in liver cells [33]. Glucokinase, a hexokinase isozyme, is a key regulator of glucose disposal and storage, and responds to increases in circulating glucose concentration by initiating a signalling cascade that results in insulin secretion from pancreatic islets $\beta$ cell [34]. Alterations in glucokinase expression and activity are associated with poorly controlled T2DM [35] and nonalcoholic fatty liver disease (NAFLD) [36]. It has been reported that common variants in the GCKR gene are associated with increased blood triglycerides [37, 38], lower fasting glucose [38], and NAFLD [39]. The glucokinase regulator protein, which binds with glucokinase and inactivates it from carbohydrate metabolism, is able to serve as a new treatment target for T2DM [40]. Significantly lower levels of GCKR mRNA in the SHS individuals in this study indicate that disorders of glucose metabolism might play an important role in the pathophysiology of SHS. Given the findings, GCKR mRNA might be a potential predictive diagnostic biomarker for the progression of SHS towards T2DM, and glucokinase regulator protein could be applied as potential therapeutic or preventive targets for SHS and T2DM.

Functional annotation and pathway enrichment analysis of DEGs provided an intuitive overview of the mechanism of SHS. The significant GO terms were BMPs signalling pathway and regulation of ERK $1 / 2$ cascade. BMPs, a group of signalling molecules, are part of the transforming growth factor- $\beta$ superfamily 
of proteins. Initially discovered for their ability to induce bone formation [41], BMPs are now known to play important roles in the adult vascular endothelium, promoting angiogenesis and mediating oxidative stress [42]. Due to the critical roles of BMPs in maintenance of adult tissue homeostasis, it is found that dysregulation in BMPs signalling pathway contribute to various diseases, including cancer, skeletal disorders and CVD [43]. Our previous studies suggested that SHS might precede the occurrence of CVD [6, 7]. In the present study, significantly lower levels of BMPER and hemojuvelin BMP co-receptor (HJV) mRNA, which involved in the BMPs signalling pathway, were found in individuals with SHS. Our findings indicate that BMPs signalling pathway may play an important role in the pathophysiology of SHS, and transcripts of BMPER and HJV could be potential diagnosis biomarkers for SHS.

ERK1 and ERK2 cascade is key signalling pathway that regulates a large variety of cellular processes, including adhesion, migration, differentiation, metabolism, and proliferation [44]. This signalling cascade is dysregulated in a variety of diseases including CVD [45], insulin resistance [46], and inflammation [47]. In current study significantly lower levels of metallothionein 3 (MT3) and C-C motif chemokine ligand 3 (CCL3) mRNA in SHS individuals indicates that the ERK1 and ERK2 cascade is associated with the progression of SHS towards chronic disease, such as CVD and T2DM.

The KEGG enrichment analysis revealed that $A B C$ transporter and neurodegeneration are the biological pathways related to SHS. ABC transporters are a large family of transmembrane proteins. These proteins bind ATP and use the energy to drive the transport of various molecules across cell membranes [48]. In human, the $48 A B C$ proteins are divided into seven subfamilies, from $A$ to $G$, based on sequence and organization of their ATP-binding domain [48]. The ABCA4 protein transports vitamin A derivatives and perform a crucial role in the visual cycle [49]. In the present study, the downregulation of $A B C A 4$ was observed in the individuals with SHS, which indicates that the decreased level of $A B C A 4$ mRNA is associated with the SHS phenotype of eye, such as eye ache and fatigue.

The ABCG8 protein functions to facilitate the transport of sterols in the intestine and liver [50]. Our previous study found that steroid hormone biosynthesis pathway is disturbed in SHS individuals [16], which indicates that the upregulation of $A B C G 8$ might be associated with the disorder of steroid hormone biosynthesis in SHS individuals. Chen and colleagues have observed that trimethylamine-N-oxide, a metabolite produced by gut microbiota, is associated with increased ABCG8 expression [51]. In addition, Zhu et al. has proved that intestinal microbiota, Enterococcus faecalis, increase the expression of ABCG8 [52]. Our previous study has found that alterations of intestinal microbiota occur in SHS individuals [15]. In the present study, the higher level of $A B C G 8$ mRNA might be associated with the diversity of intestinal microbiota in SHS individuals. Significantly lower levels of TUBB3 and calcium/calmodulin dependent protein kinase II $\beta$ (CAMK2B) mRNA, which involved in the Parkinson's disease and neurodegeneration, were observed in SHS individuals. These findings indicate that neurodegeneration might be involved in the pathophysiology of SHS.

The PPI network enables the exploration and visualization of functional interactions between the DEGs. As shown in Fig. 5, GJA1, TWIST2, KRT1, TUBB3, AMHR2, BMP10, MT3, BMPER, NTM and TMEM98, were 
identified and selected as critical hub genes. Gap junction protein a1 (GJA1), also known as connexin 43 protein, is protein subunit that constitute gap junction channels [53]. The intercellular channels of gap junction facilitate the transfer of ions and small molecular from cell to cell, and are thought to modulate several processes, including embryogenesis, differentiation, and electrotonic coupling [54]. GJA1 expression is affected by several pathophysiological conditions, such as hypertension, hypercholesterolemia, and diabetes [55]. In the present study, the higher level of GJA1 mRNA in SHS individuals indicates that GJA1 mRNA could be associated with the progression of SHS phenotype towards CVD and T2DM. In addition, Squecco et al. has reported that the bioactive sphingolipid, sphingosine 1-phosphate, can enhance GJA1 protein expression [56]. Our previous study has found sphingolipids metabolism is the disturbed metabolic pathway related to SHS, and significantly higher levels of sphinganine 1-phosphate and sphingomyelin are observed in SHS individuals [16]. Given these findings, the upregulated GJA1 mRNA could be affected by the disturbed sphingolipids metabolism in SHS individuals. These critical genes play hub roles in predictive, preventive, and personalized medicine related to SHS, and be worthy of further investigation.

To establish a relatively accurate diagnosis model for individuals with SHS, a logistic regression analysis was performed based on the transcripts of 10 identified hub genes. ROC curve analysis showed that the predictive diagnosis model based on transcriptomic biomarkers can distinguish individuals with SHS from individuals with ideal health status with a sensitivity of $83.3 \%$, a specificity of $90.0 \%$, and an AUC of 0.938. These findings exhibit strong predictive abilities of transcriptomic biomarkers for SHS diagnosis. Blood transcripts are potentially objective biomarkers for the SHS diagnosis. The proposed transcriptomic biomarkers have a promising prospect of clinical application in the prediction and prevention of chronic disease.

To the best of our knowledge, this is the first study to screen transcriptomic biomarkers for SHS using RNA-Seq-based transcriptome profiling. Nevertheless, several limitations in the present study are noteworthy. First, our study is a case-control study with a relatively small sample size, hence the generalisation of these findings could be questioned. However, considering the fact that our study provides the original observations on the transcriptomic features of SHS population, the present study has provided a new idea that buffy coat transcripts might offer a novel alternative for the predictive diagnosis, targeted prevention and personalized treatment of chronic diseases. In addition, considering the quantitative accuracy of RNA-Seq technology, a RT-qPCR study is underway against the same cohort to validate the putative transcript biomarkers and selected hub genes based on the findings in this study. Building on the present findings, further studies of larger cohorts from diverse geographical areas and populations with different age ranges are warranted.

\section{Conclusions And Expert Recommendations}

The early diagnosis of SHS has the potential of predicting chronic diseases at early stage, and effective intervention on SHS may be a cost-effective way for the targeted prevention, and personalized therapy of chronic diseases. For the first time, the present study identified 46 DEGs between the SHS individuals and 
healthy controls using RNA-Seq-based transcriptome profiling. A total of 23 transcripts was selected as candidate diagnostic biomarkers for SHS. The present study clearly revealed the potential value of transcriptomic biomarkers for the predictive diagnosis of SHS from the perspective of PPPM. The pattern of the differentially expressed genes can be used as biomarkers for patient stratification. We suggest that SHS-related transcriptomic biomarkers and other SHS-related biomarkers at multi-omics levels are the key promise for the practice of PPPM of chronic disease.

The downregulation of GCKR in SHS individuals indicated that glucose metabolism disorder plays a significant role in the pathophysiologic mechanism of SHS. The GCKR protein could serve as a potentially preventive/therapeutic target for the progression from SHS towards T2DM. We recommend that further studies of large prospective cohorts should be conducted to investigate the crucial role of $G C K R$ in the SHS progression, which could be useful for the predictive diagnosis and targeted prevention of T2DM.

The present study demonstrated that BMPs signalling pathway, ERK $1 / 2$ cascade and $A B C$ transporters, play potential roles in the pathophysiologic mechanism of SHS. These findings determined the potential utility of SHS-related signalling pathways for targeted prevention and personalized therapy of chronic diseases. We recommend strengthening the studies of signaling pathways in SHS with different omics strategies. Ten genes, including GJA1, TWIST2, KRT1, TUBB3, AMHR2, BMP10, MT3, BMPER, NTM and TMEM98, play hub roles in the underlying mechanisms of SHS. We also suggest that it is crucial to deeply study the functions and activities of these differentially expressed genes in the underly mechanism of SHS.

For the first time, the present study found the association between neurodegeneration and SHS. These findings indicated that SHS may precede the actual onset of neurodegeneration diseases, such as Alzheimer's disease and Parkinson's disease. The integration of subjective health measure (SHSQ-25) and objective biomarkers (transcripts of TUBB3 and $C A M K 2 B$ ) enables clinician and public health workers to predict individual's high risk of developing neurodegeneration diseases. SHSQ-25 can be used as an alternative health screening tool in the population-based health survey, particularly when it is lack of laboratory-based resources. Primary healthcare providers must be able to detect and manage SHS to fight delayed diagnosis, untargeted prevention, and ineffective intervention of chronic disease.

\section{Declarations}

\section{Acknowledgments}

The authors acknowledge the participants and their families who donated their time and effort in helping to make this study possible.

\section{Authors' contribution}


HW and YW participated in the design of the study. HW, QT, JZ, HL, WC, XZ, XL, LW, MS and YK performed participant enrollment and collected the samples. HW, QT and JXZ performed the Transcriptome analysis. HW and QT performed the statistical analysis and drafted the manuscript. YK, WW and YW revised the manuscript.

\section{Funding information}

This work was partially supported by the "Hundred-Thousand-Ten Thousand Project (2020A17)" and the National Natural Science Foundation of China (Grant Numbers: 81872682 \& 81773527). HW was supported by the China Scholarship Council (CSC 201708110200).

\section{Compliance and Ethical Standards}

\section{Conflicts of interest}

The authors confirm that there are no conflicts of interest.

\section{Consent for publication}

Not applicable

\section{Ethical approval and consent to participate}

This study was approved by the Ethics Committee of the Weifang University, Weifang, China. Written informed consent was obtained from each participant at the beginning of the study. The ethics approval was given in compliance with the Declaration of Helsinki.

\section{Abbreviations}

$A B C$, ATP-binding cassette; AUC, area under the curve; BMPs, bone morphogenetic proteins; BP, biological process; CC, cellular component; CDNA, complementary DNA; Cl, confidence interval; CVD, cardiovascular disease; DEGs, differentially expressed genes; ERK1, extracellular signal-regulated kinase-1; FDR, false discovery rate; GO, gene ontology; IQR, interquartile ranges; KEGG, kyoto encyclopedia of genes and genomes; MF, molecular function; NAFLD, nonalcoholic fatty liver disease; PPI, protein-protein Interaction; PPPM, preventive, predictive and personalised medicine; RNA-Seq, RNA sequencing; ROC, receiver operating characteristic; SD, standard deviation; SHS, suboptimal health status; SHSQ-25, suboptimal health status questionnaire-25; STRING, search tool for the retrieval of interacting genes; T2DM, type 2 diabetes mellitus.

\section{References}

1. Golubnitschaja O, Watson ID, Topic E, Sandberg S, Ferrari M, Costigliola V. Position paper of the EPMA and EFLM: a global vision of the consolidated promotion of an integrative medical approach 
to advance health care. EPMA J. 2013;4(1):12.

2. Golubnitschaja O, Baban B, Boniolo G, Wang W, Bubnov R, Kapalla M, et al. Medicine in the early twenty-first century: paradigm and anticipation - EPMA position paper 2016. EPMA J. 2016;7:23.

3. Golubnitschaja O, Costigliola V. Epma. General report \& recommendations in predictive, preventive and personalised medicine 2012: white paper of the European Association for Predictive, Preventive and Personalised Medicine. EPMA J. 2012;3(1):14.

4. Adua E, Roberts $P$, Wang W. Incorporation of suboptimal health status as a potential risk assessment for type II diabetes mellitus: a case-control study in a Ghanaian population. EPMA J. 2017;8(4):34555.

5. Ge S, Xu X, Zhang J, Hou H, Wang H, Liu D, et al. Suboptimal health status as an independent risk factor for type 2 diabetes mellitus in a community-based cohort: the China suboptimal health cohort study. Epma Journal. 2019;10(1):1-8.

6. Yan Y, Dong J, Liu Y, Yang X, Li M, Shia G, et al. Association of suboptimal health status and cardiovascular risk factors in urban Chinese workers. J Urban Health. 2012;89(2):329-38.

7. Wang Y, Liu X, Qiu J, Wang H, Liu D, Zhao Z, et al. Association between Ideal Cardiovascular Health Metrics and Suboptimal Health Status in Chinese Population. Sci Rep. 2017;7(1):14975.

8. Wang W, Yan Y. Suboptimal health: a new health dimension for translational medicine. Clin Transl Med. 2012;1(1):28.

9. Yan Y, Liu Y, Li M, Hu P, Guo A, Yang X, et al. Development and evaluation of a questionnaire for measuring suboptimal health status in urban Chinese. J Epidemiol. 2009;19(6):333-41.

10. Kupaev V, Borisov O, Marutina E, Yan Y, Wang W. Integration of suboptimal health status and endothelial dysfunction as a new aspect for risk evaluation of cardiovascular disease. EPMA J. 2016;7(1):19.

11. Anto EO, Roberts P, Coall D, Turpin CA, Adua E, Wang Y, et al. Integration of suboptimal health status evaluation as a criterion for prediction of preeclampsia is strongly recommended for healthcare management in pregnancy: a prospective cohort study in a Ghanaian population. EPMA J. 2019;10(3):211-26.

12. Wang Y, Ge S, Yan Y, Wang A, Zhao Z, Yu X, et al. China suboptimal health cohort study: rationale, design and baseline characteristics. J Transl Med. 2016;14(1):291.

13. Yan Y, Wu L, Xiao H, Wang S, Dong J, Wang W. Latent class analysis to evaluate performance of plasma cortisol, plasma catecholamines, and SHSQ-25 for early recognition of suboptimal health status. EPMA J. 2018;9(3):299-305.

14. Alzain MA, Asweto CO, Zhang J, Fang H, Zhao Z, Guo X, et al. Telomere Length and Accelerated Biological Aging in the China Suboptimal Health Cohort: A Case-Control Study. OMICS. 2017;21(6):333-9.

15. Sun Q, Xu X, Zhang J, Sun M, Tian Q, Li Q, et al. Association of suboptimal health status with intestinal microbiota in Chinese youths. J Cell Mol Med. 2019;24(2):1837-47. 
16. Wang H, Tian QY, Zhang J, Liu HQ, Zhang XY, Cao WJ, et al. Population-based case-control study revealed metabolomic biomarkers of suboptimal health status in Chinese population-potential utility for innovative approach by predictive, preventive, and personalized medicine. EPMA J. 2020;11(2):147-60.

17. Byron SA, Van Keuren-Jensen KR, Engelthaler DM, Carpten JD, Craig DW. Translating RNA sequencing into clinical diagnostics: opportunities and challenges. Nat Rev Genet. 2016;17(5):25771.

18. Wang Z, Gerstein M, Snyder M. RNA-Seq: a revolutionary tool for transcriptomics. Nat Rev Genet. 2009;10(1):57-63.

19. Casamassimi A, Federico A, Rienzo M, Esposito S, Ciccodicola A. Transcriptome Profiling in Human Diseases: New Advances and Perspectives. Int J Mol Sci. 2017;18(8):1652.

20. Yan Y, Dong J, Liu Y, Zhang J, Song M, He Y, et al. Association of suboptimal health status with psychosocial stress, plasma cortisol and mRNA expression of glucocorticoid receptor alpha/beta in lymphocyte. Stress. 2015;18(1):29-34.

21. Sweetman E, Ryan M, Edgar C, MacKay A, Vallings R, Tate W. Changes in the transcriptome of circulating immune cells of a New Zealand cohort with myalgic encephalomyelitis/chronic fatigue syndrome. Int J Immunopathol Pharmacol. 2019;33:2058738418820402.

22. Tomas-Roig J, Havemann-Reinecke U. Gene expression signature in brain regions exposed to longterm psychosocial stress following acute challenge with cannabinoid drugs.

Psychoneuroendocrinology. 2019;102:1-8.

23. Craig CL, Marshall AL, Sjostrom M, Bauman AE, Booth ML, Ainsworth BE, et al. International physical activity questionnaire: 12-country reliability and validity. Med Sci Sports Exerc. 2003;35(8):1381-95.

24. Soldatos CR, Dikeos DG, Paparrigopoulos TJ. Athens Insomnia Scale: validation of an instrument based on ICD-10 criteria. J Psychosom Res. 2000;48(6):555-60.

25. Shear MK, Vander Bilt J, Rucci P, Endicott J, Lydiard B, Otto MW, et al. Reliability and validity of a structured interview guide for the Hamilton Anxiety Rating Scale (SIGH-A). Depress Anxiety. 2001;13(4):166-78.

26. Bagby RM, Ryder AG, Schuller DR, Marshall MB. The Hamilton Depression Rating Scale: has the gold standard become a lead weight? Am J Psychiatry. 2004;161(12):2163-77.

27. Liao Y, Smyth GK, Shi W. featureCounts: an efficient general purpose program for assigning sequence reads to genomic features. Bioinformatics. 2014;30(7):923-30.

28. Love MI, Huber W, Anders S. Moderated estimation of fold change and dispersion for RNA-seq data with DESeq2. Genome Biol. 2014;15(12):550.

29. Yu G, Wang LG, Han Y, He QY. clusterProfiler: an R package for comparing biological themes among gene clusters. OMICS. 2012;16(5):284-7.

30. von Mering C, Huynen M, Jaeggi D, Schmidt S, Bork P, Snel B. STRING: a database of predicted functional associations between proteins. Nucleic Acids Res. 2003;31(1):258-61. 
31. Chin $\mathrm{CH}$, Chen $\mathrm{SH}, \mathrm{Wu} \mathrm{HH}, \mathrm{Ho} \mathrm{CW}$, Ko MT, Lin CY. cytoHubba: identifying hub objects and subnetworks from complex interactome. BMC Syst Biol. 2014;8(Suppl 4):11.

32. R Foundation for Statistical Computing. R: A language and environment for statistical computing. 2017. https://www.R-project. org/. Accessed 19 Jan 2021.

33. Van Schaftingen E. A protein from rat liver confers to glucokinase the property of being antagonistically regulated by fructose 6-phosphate and fructose 1-phosphate. Eur J Biochem. 1989;179(1):179-84.

34. Agius L. Glucokinase and molecular aspects of liver glycogen metabolism. Biochem J. 2008;414(1):1-18.

35. Haeusler RA, Camastra S, Astiarraga B, Nannipieri M, Anselmino M, Ferrannini E. Decreased expression of hepatic glucokinase in type 2 diabetes. Mol Metab. 2015;4(3):222-6.

36. Peter A, Stefan N, Cegan A, Walenta M, Wagner S, Konigsrainer A, et al. Hepatic glucokinase expression is associated with lipogenesis and fatty liver in humans. J Clin Endocrinol Metab. 2011;96(7):E1126-30.

37. Orho-Melander M, Melander O, Guiducci C, Perez-Martinez P, Corella D, Roos C, et al. Common missense variant in the glucokinase regulatory protein gene is associated with increased plasma triglyceride and C-reactive protein but lower fasting glucose concentrations. Diabetes. 2008;57(11):3112-21.

38. Diabetes Genetics Initiative of Broad Institute of H. Mit LU, Novartis Institutes of BioMedical Saxena R, Voight R, Lyssenko BF V et al. Genome-wide association analysis identifies loci for type 2 diabetes and triglyceride levels. Science. 2007;316(5829):1331-6.

39. Zain SM, Mohamed Z, Mohamed R. Common variant in the glucokinase regulatory gene rs780094 and risk of nonalcoholic fatty liver disease: a meta-analysis. J Gastroenterol Hepatol. 2015;30(1):21-7.

40. Kaushik A, Kaushik M. Recent Updates on Glucokinase Activators and Glucokinase Regulatory Protein Disrupters for the Treatment of Type 2 Diabetes Mellitus. Curr Diabetes Rev. 2019;15(3):20512.

41. Urist MR. Bone: formation by autoinduction. Science. 1965;150(3698):893-9.

42. Dyer LA, Pi X, Patterson C. The role of BMPs in endothelial cell function and dysfunction. Trends Endocrinol Metab. 2014;25(9):472-80.

43. Wang RN, Green J, Wang Z, Deng Y, Qiao M, Peabody M, et al. Bone Morphogenetic Protein (BMP) signaling in development and human diseases. Genes Dis. 2014;1(1):87-105.

44. Roskoski R Jr. ERK1/2 MAP kinases: structure, function, and regulation. Pharmacol Res. 2012;66(2):105-43.

45. Muslin AJ. MAPK signalling in cardiovascular health and disease: molecular mechanisms and therapeutic targets. Clin Sci (Lond). 2008;115(7):203-18. 
46. Tanti JF, Jager J. Cellular mechanisms of insulin resistance: role of stress-regulated serine kinases and insulin receptor substrates (IRS) serine phosphorylation. Curr Opin Pharmacol. 2009;9(6):75362.

47. Kim EK, Choi EJ. Pathological roles of MAPK signaling pathways in human diseases. Biochim Biophys Acta. 2010;1802(4):396-405.

48. Higgins CF. ABC transporters: from microorganisms to man. Annu Rev Cell Biol. 1992;8:67-113.

49. Sun H, Molday RS, Nathans J. Retinal stimulates ATP hydrolysis by purified and reconstituted ABCR, the photoreceptor-specific ATP-binding cassette transporter responsible for Stargardt disease. J Biol Chem. 1999;274(12):8269-81.

50. Berge KE, Tian H, Graf GA, Yu L, Grishin NV, Schultz J, et al. Accumulation of dietary cholesterol in sitosterolemia caused by mutations in adjacent ABC transporters. Science. 2000;290(5497):1771-5.

51. Chen Y, Weng Z, Liu Q, Shao W, Guo W, Chen C, et al. FMO3 and its metabolite TMAO contribute to the formation of gallstones. Biochim Biophys Acta Mol Basis Dis. 2019;1865(10):2576-85.

52. Zhu Y, Li T, Din AU, Hassan A, Wang Y, Wang G. Beneficial effects of Enterococcus faecalis in hypercholesterolemic mice on cholesterol transportation and gut microbiota. Appl Microbiol Biotechnol. 2019;103(7):3181-91.

53. Fishman GI, Eddy RL, Shows TB, Rosenthal L, Leinwand LA. The human connexin gene family of gap junction proteins: distinct chromosomal locations but similar structures. Genomics. 1991;10(1):2506.

54. Beyer EC, Paul DL, Goodenough DA. Connexin family of gap junction proteins. J Membr Biol. 1990;116(3):187-94.

55. Schulz R, Gorge PM, Gorbe A, Ferdinandy P, Lampe PD, Leybaert L. Connexin 43 is an emerging therapeutic target in ischemia/reperfusion injury, cardioprotection and neuroprotection. Pharmacol Ther. 2015;153:90-106.

56. Squecco R, Sassoli C, Nuti F, Martinesi M, Chellini F, Nosi D, et al. Sphingosine 1-phosphate induces myoblast differentiation through $\mathrm{C} \times 43$ protein expression: a role for a gap junction-dependent and independent function. Mol Biol Cell. 2006;17(11):4896-910.

\section{Figures}




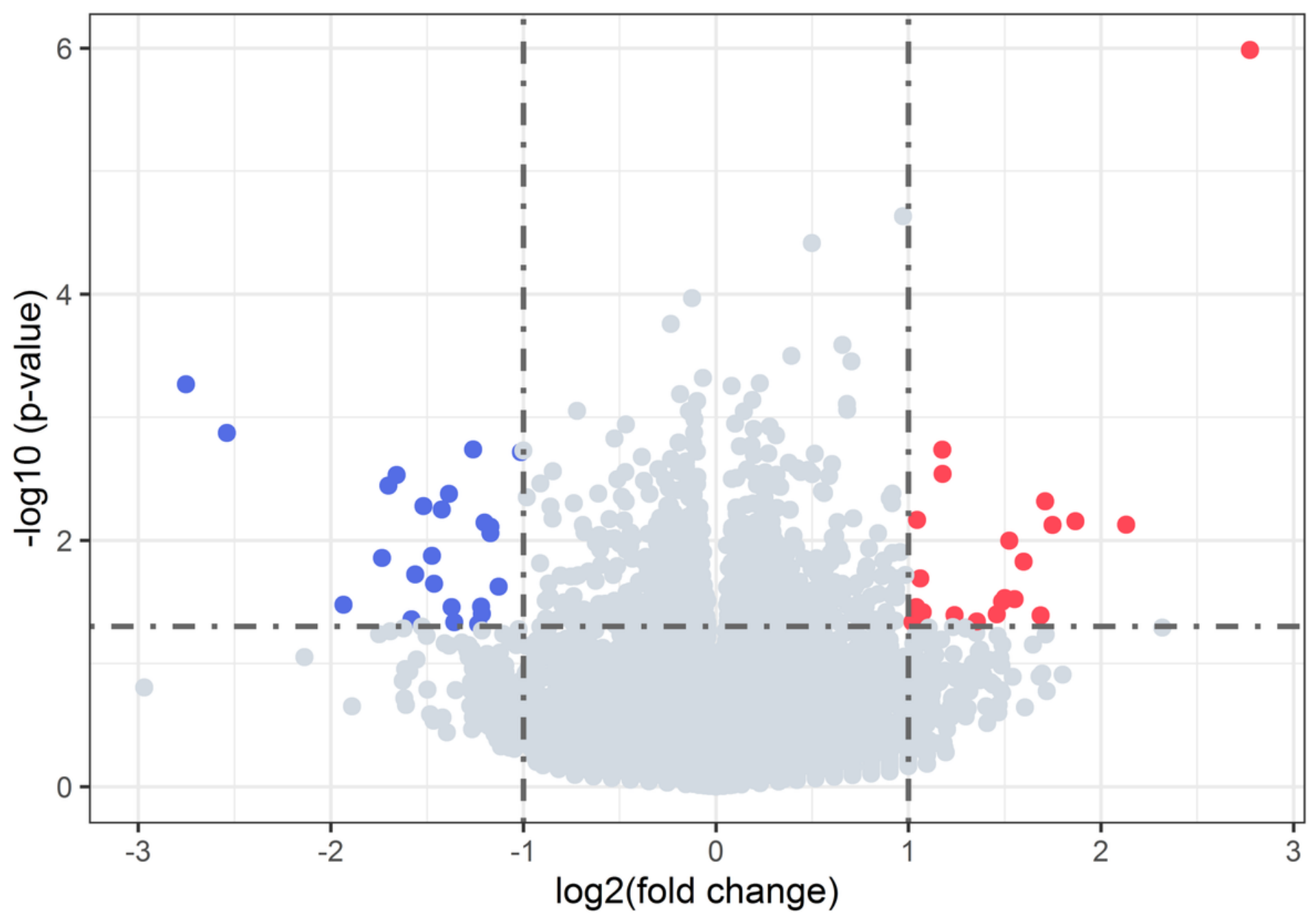

- Down Stable $\bullet$ Up

Figure 1

Volcano plot of DEGs Note: The upregulated genes are presented by red color, while the downregulated genes are presented by blue color; DEGs, differential expressed genes. 


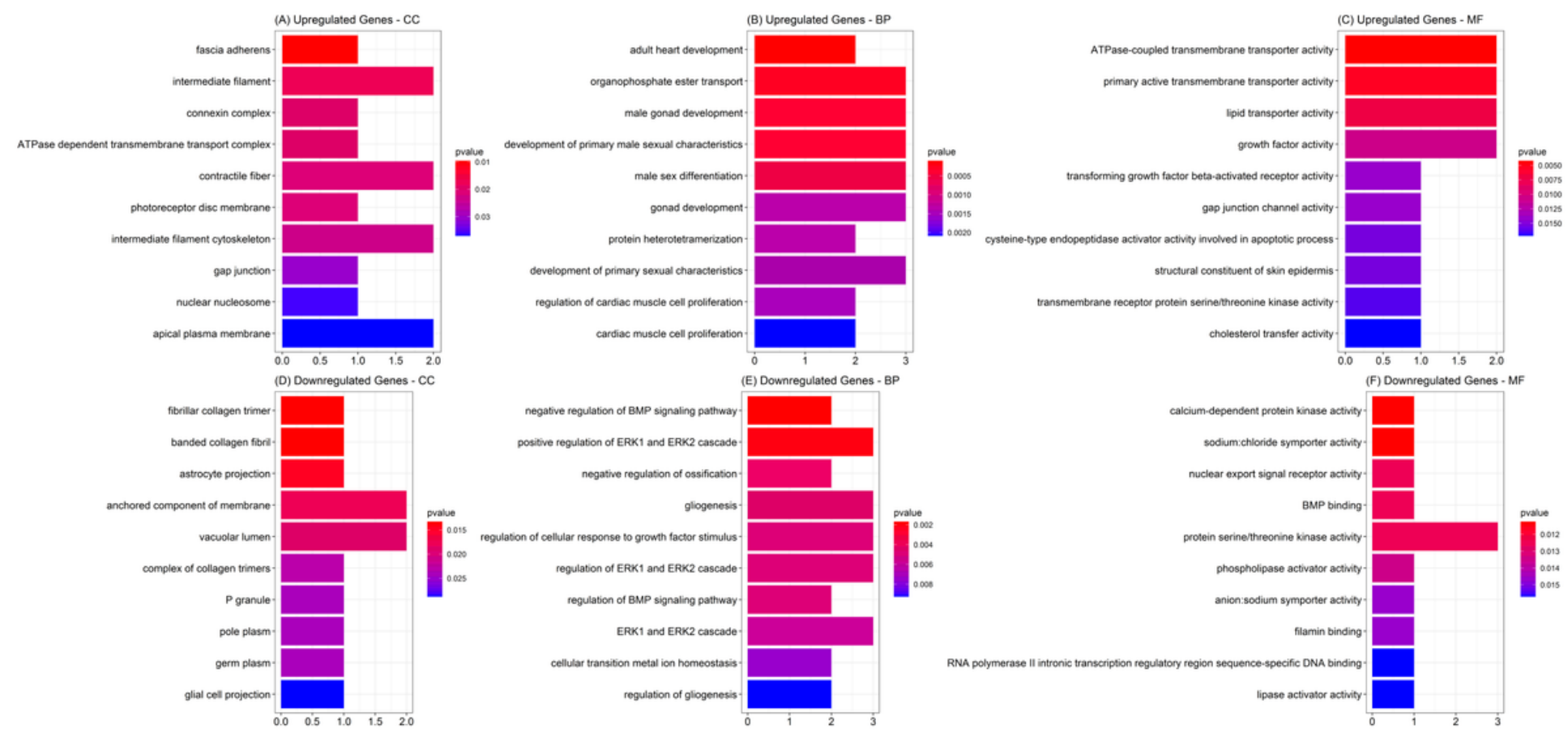

Figure 2

GO enrichment analysis of DEGs. Note: BP, biological process; CC, cellular component; MF molecular function; GO, Gene ontology; DEGs, differentially expressed genes. The y-axis represents $\mathrm{GO}$ enriched terms. The $x$-axis represents the gene ratio under specific GO terms. The color of the bar represents the $P$ value.
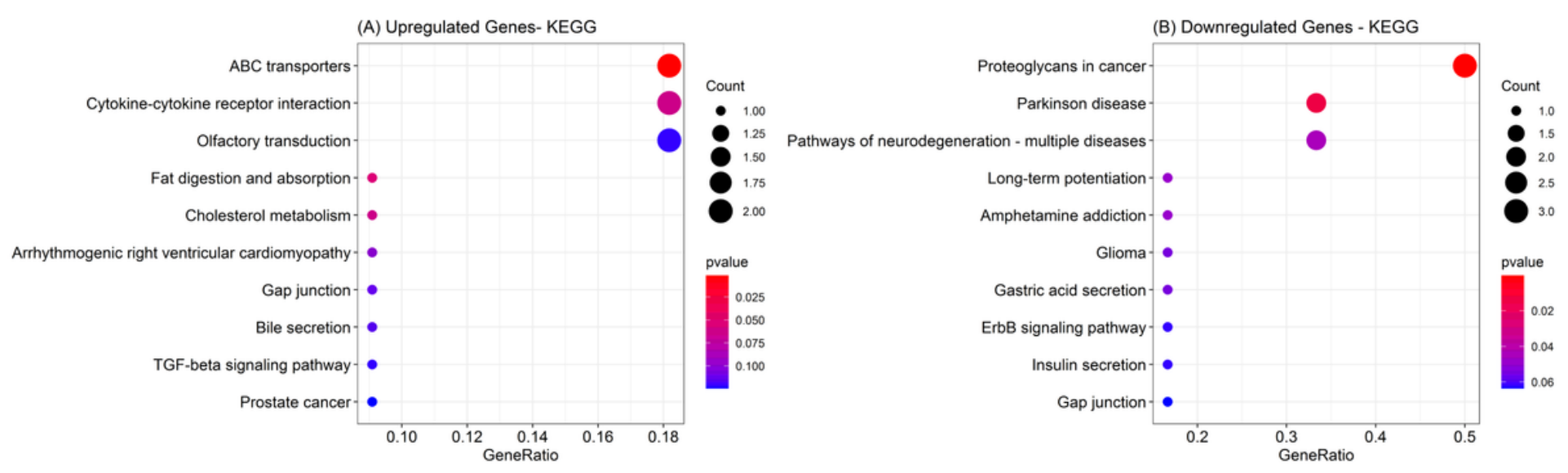

\section{Figure 3}

KEGG enrichment analysis of DGEs Note: KEGG, Kyoto Encyclopedia of Genes and Genomes; DEGs, differentially expressed genes. The y-axis represents KEGG enriched terms. The $x$-axis represents the gene ratio under a specific KEGG terms. The color of the bar represents the $P$ value. The size of dot represents the count of genes under specific KEGG terms. 


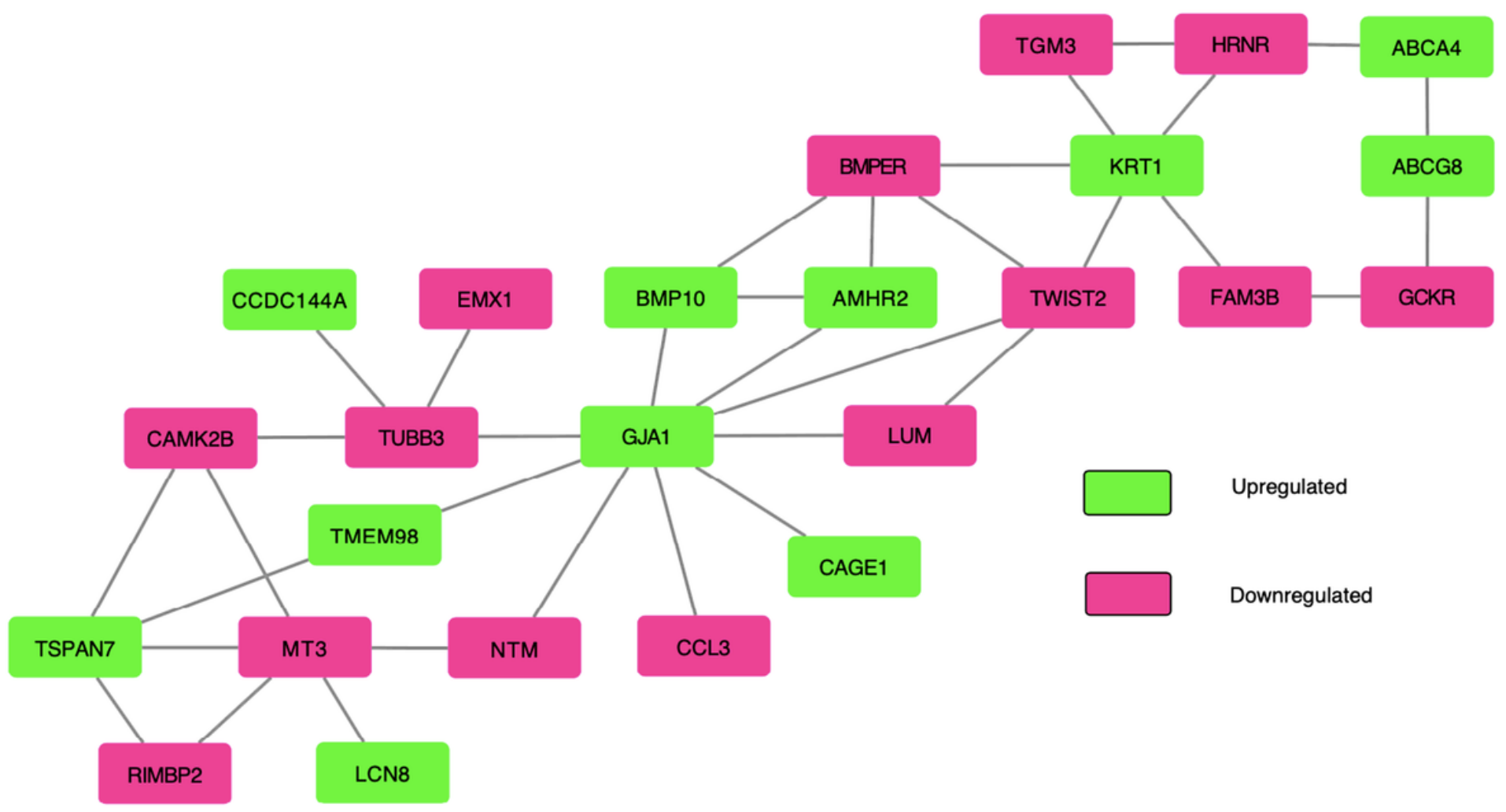

Figure 4

PPI network of DEGs Note: DEGs, differentially expressed genes. PPI, protein-protein interaction. The nodes represent the significantly upregulated/downregulated genes. The edges represent the interaction of proteins coded by DEGs. The resource for the abbreviations of the protein names is form the STRING database; STRING, Search Tool for the Retrieval of Interacting Genes. 


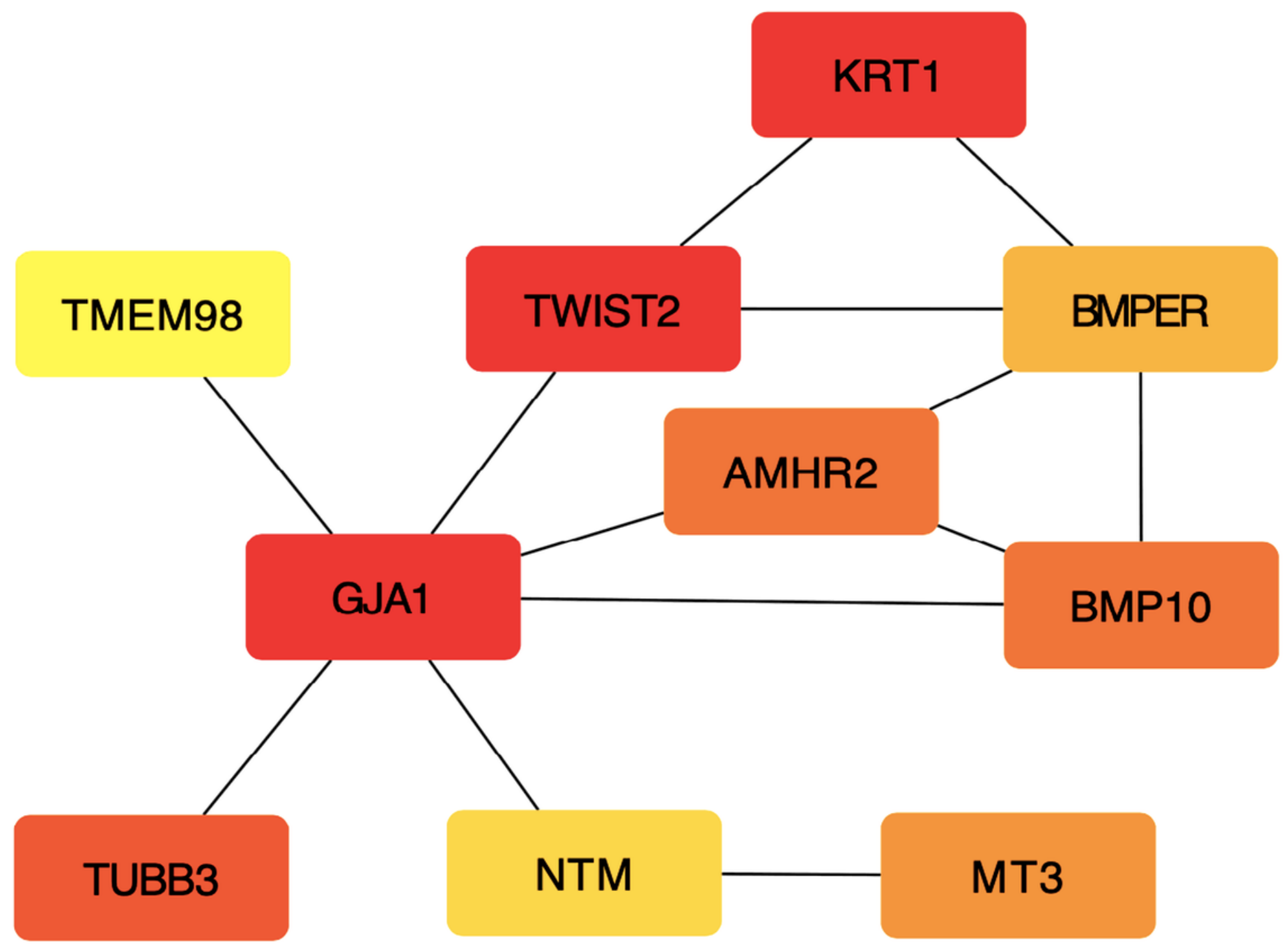

Figure 5

Hub genes identified in PPI network Note: PPI, protein-protein interaction; The nodes represent the significantly upregulated/downregulated genes. The edges represent the interaction of proteins coded by DEGs. The node color changes gradually from yellow to red in ascending order according to the degree of the genes. The resource for the abbreviations of the protein names is form the STRING database; STRING, Search Tool for the Retrieval of Interacting Genes. 


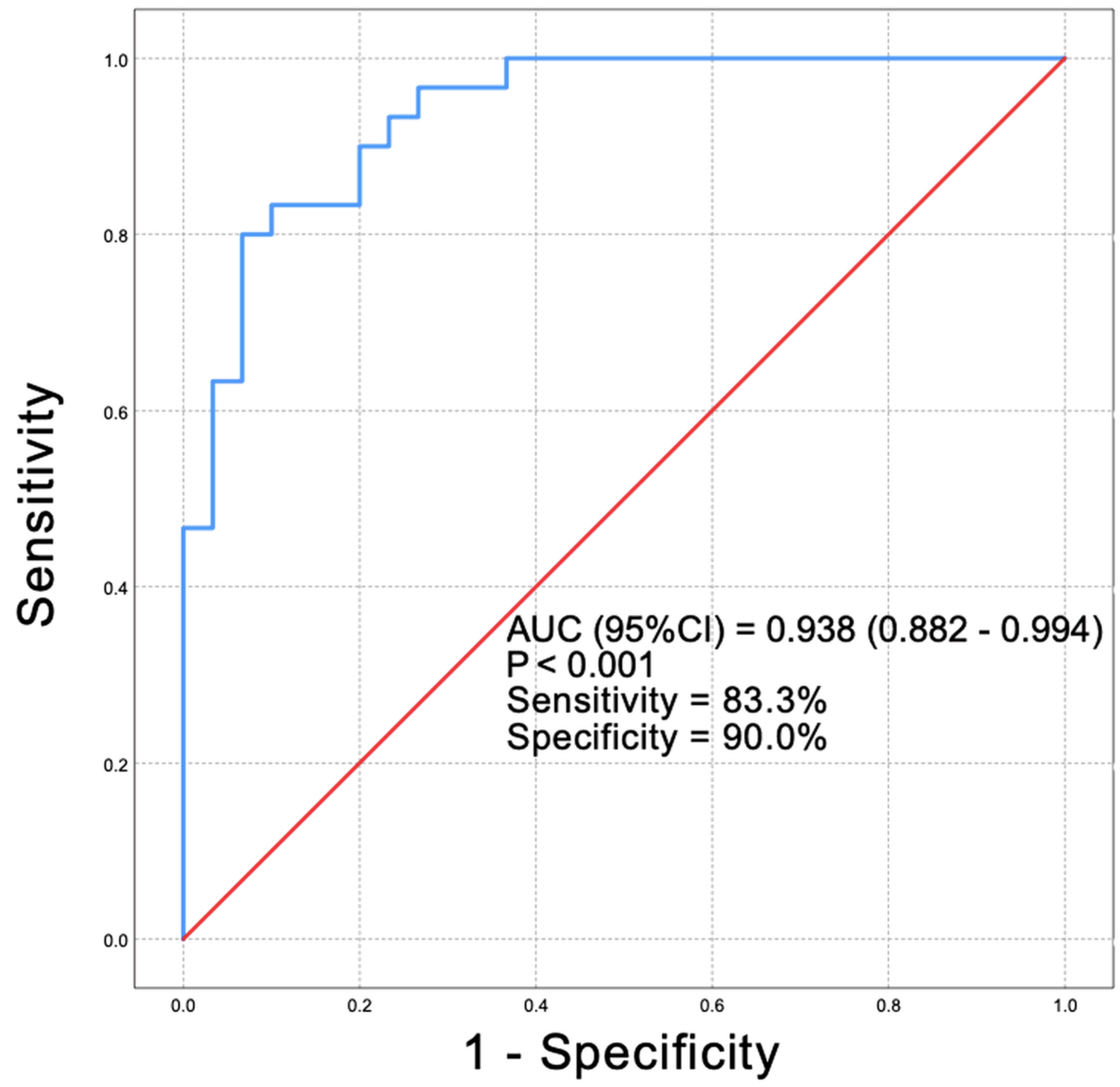

Figure 6

ROC curve for transcriptome classification model Note: AUC, area under the curve; $\mathrm{Cl}$, confidence interval; $\mathrm{ROC}$, receiver operating characteristic.

\section{Supplementary Files}

This is a list of supplementary files associated with this preprint. Click to download.

- SupplementaryTables.pdf 